\title{
Santé mentale et douleur
}

Composantes somatiques et psychiatriques

de la douleur en santé mentale 


\section{Springer}

Paris

Berlin

Heidelberg

New York

Hong Kong

Londres

Milan

Tokyo 
Santé mentale et douleur

Composantes somatiques

et psychiatriques

de la douleur en santé mentale

望 Springer 


\title{
Sous la direction de :
}

\section{Serge Marchand}

Centre de recherche clinique Étienne-Le Bel du CHUS

Université de Sherbrooke, Faculté de médecine

Département de chirurgie - Service de neurochirurgie

3001, $12^{\mathrm{e}}$ Avenue Nord

Sherbrooke (Québec) Canada J1H 5N4

\section{Djea Saravane}

Hôpital de Ville-Evrard

Service des spécialités et de médecine polyvalente

202, avenue Jean-Jaurès

93330 Neuilly-sur-Marne

\section{Isabelle Gaumond}

Centre de recherche clinique Étienne-Le-Bel du CHUS

3001, $12^{\mathrm{e}}$ Avenue Nord

Sherbrooke (Québec) Canada J1H 5N4

ISBN : 978-2-8178-0306-7 Springer Paris Berlin Heidelberg New York

(c) Springer-Verlag France, Paris, 2013

\begin{abstract}
Cet ouvrage est soumis au copyright. Tous droits réservés, notamment la reproduction et la représentation, la traduction, la réimpression, l'exposé, la reproduction des illustrations et des tableaux, la transmission par voie d'enregistrement sonore ou visuel, la reproduction par microfilm ou tout autre moyen ainsi que la conservation des banques de données. La loi française sur le copyright du 9 septembre 1965 dans la version en vigueur n'autorise une reproduction intégrale ou partielle que dans certains cas, et en principe moyennant le paiement des droits. Toute représentation, reproduction, contrefaçon ou conservation dans une banque de données par quelque procédé que ce soit est sanctionnée par la loi pénale sur le copyright.
\end{abstract}

L'utilisation dans cet ouvrage de désignations, dénominations commerciales, marques de fabrique, etc. même sans spécification ne signifie pas que ces termes soient libres de la législation sur les marques de fabrique et la protection des marques et qu'ils puissent être utilisés par chacun.

La maison d'édition décline toute responsabilité quant à l'exactitude des indications de dosage et des modes d'emplois. Dans chaque cas il incombe à l'usager de vérifier les informations données par comparaison à la littérature existante.

Maquette de couverture : Nadia Ouddane

Mise en page : Desk

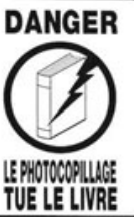

Illustration de couverture : (c) Eky Chan - Fotolia.com 
Préface

A. Damasio

Introduction

1. La douleur en santé mentale : les mythes.

D. Saravane

2. Bases neurophysiologiques de la douleur

S. Marchand

3. Perception de la douleur en santé mentale

Ph. Goffaux, G. Léonard, M. Lévesque

4. Mosaïque épidémiologique de la douleur chronique en santé mentale : l'exemple de la dépression

A. Vanasse, M. Courteau, J. Courteau, N. Carrier

5. La douleur dans la dépression majeure :

de l'évidence clinique au paradoxe expérimental

S. Potvin

6. Dépression et douleur : approche clinique

E. Corruble

7. Trouble bipolaire et douleur : au-delà de la souffrance

É. Olié, P. Courtet

8. Douleur et schizophrénie : quand l'esprit ignore les appels grandissants de la moelle 
9. Schizophrénie et douleur : une perspective psychiatrique

S. Grignon

10. Troubles somatoformes et douleur

P. Cailliez, P. Hardy

11. Douleur et état de stress post-traumatique

A. Yrondi, J.-F. Corbin, L. Schmitt

12. Douleur chez l'enfant autiste

T.F. Oberlander, L. Zeltzer

13. Opioïdes, douleur et toxicomanie : plus de peur que de mal ?

K. Stavro, S. Potvin

14. Moyens d'évaluation de la douleur en santé mentale..

D. Saravane, A. Serrie

15. Prise en charge et perspectives thérapeutiques.

D. Saravane 


\section{Préface}

\section{Longtemps après la douleur}

Il est difficile d'exagérer l'importance de la douleur. Chaque lecteur de ce livre aura déjà subi la douleur, à un moment ou un autre, légère ou grave, importune et incommodante, voire même perturbatrice. Tout lecteur de ce livre aura également entendu parler de personnes en douleur et aura été témoin des effets de la douleur sur leur comportement. Quiconque présentant une intelligence raisonnable et des émotions sensées n'aura pas cherché à connaître la douleur et aura certainement essayé de l'éviter. Toutefois, ironie du sort, ce phénomène omniprésent fait partie de la vie normale, et ce, malgré l'expérience négative associée à la douleur et sa signification souvent inquiétante.

Alors, comment peut-on donner un sens à cet invité conventionnel mais non désiré ? Quel rôle lui est attribué dans la vie des individus et dans la vie collective des espèces animales?

La réponse à la première question est plutôt évidente. La douleur est protectrice. La douleur signale le moment où l'intégrité des cellules et des tissus devient nettement menacée ou qu'elle est déjà perdue, dans un secteur du corps. Une perturbation est détectée quelque part dans un corps vivant, est signalée au système nerveux central et est localisée en fonction de ses coordonnées spatiales dans une carte neuronale associée. Mais la douleur n'est pas seulement une sentinelle indifférente, un avertisseur de fumée neutre. Elle est également un messager, transmettant l'information sur l'emplacement et la gravité du problème à un maître contrôleur. La réponse adaptative, visant à faire ce qui peut être fait pour atténuer le problème, peut commencer dès que la cartographie est réalisée, en fait, avant même que la douleur, prise au sens propre, ne commence. C'est parce que la douleur est un sentiment, une expérience subjective, et, par définition, les sentiments sont des événements mentaux qui se produisent dans la conscience. En bref, la cartographie simple d'un équilibre perturbé, la carte dite nociceptive, peut donner lieu à une réponse adaptative, même avant qu'elle ne soit ressentie comme une douleur. Si et quand elle se transforme en un sentiment, la douleur survient, et la douleur au sens propre, comme tout autre sentiment, devient une nouvelle couche de contrôle au-delà de la cartographie simple. Ce niveau expérientiel ajouté permet de nouvelles formes de réponse. 
Ainsi, le point crucial de la douleur est une sensation désagréable produite à partir d'une carte nociceptive dans certaines circonstances physiologiques. Elle produit un nouveau type d'effet à l'intérieur de l'être vivant dans lequel elle se produit, elle joue avec sa mémoire, imprègne son imagination, affecte son raisonnement, et permet d'engendrer une réponse délibérée. C'est là que réside la signification de la douleur pour toutes les espèces qui peuvent en faire l'expérience : au-delà des répertoires de réponses de routine programmées dans les systèmes nerveux, ces espèces gagnent une nouvelle raison d'exercer un contrôle sur leur vie, souvent, comme dans le cas des humains, un contrôle imaginatif et créatif. La douleur, dans toutes ses nuances, et le plaisir dans toutes ses variétés, sont les principaux arbitres de régulation de la vie et des moteurs essentiels dans l'évolution.

Cette toile de fond explique le rôle primordial que joue la douleur en médecine, ainsi que la nécessité de bien la diagnostiquer, l'interpréter et la gérer et donc l'importance de ce volume didactique, assemblé par Serge Marchand, un expert de la douleur reconnu internationalement, ainsi que ses collègues, Djéa Saravane, chef de service, praticien hospitalier et chargé d'enseignement, et Isabelle Gaumond, une biologiste médicale spécialisée en douleur. Leur livre réunit des spécialistes ayant une vision similaire du problème, en donnant une place de choix à la relation importante entre la douleur et la santé mentale. En effet, les aspects psychiatriques de conditions douloureuses représentent l'un des plus grands défis pour les scientifiques et les cliniciens. Ils sont parmi les plus difficiles à traiter. La dépression, la schizophrénie, le stress post-traumatique et l'addiction sont traités dans des chapitres distincts, et même les problèmes particuliers posés par les enfants ne sont pas négligés. Un chapitre sur la neurophysiologie de la douleur et un autre sur les aspects historiques complètent la liste.

Il s'agit d'un volume plus que nécessaire qui trouvera sa place dans la bibliothèque de tous ceux qui en ont besoin pour diagnostiquer et traiter des conditions de douleur dans le cadre de la santé mentale. Il est rempli de renseignements utiles et de conseils pratiques et mérite notre attention.

Antonio Damasio

University Professor

David Dornsife Professor of Neuroscience

Director, Brain and Creativity Institute University of Southern California 